OPEN ACCESS

Edited by:

Yong-Jing Gao,

Nantong University, China

Reviewed by:

Zhifang Dong,

Chongqing Medical University, China

Xinhua Zhang,

Nantong University, China

*Correspondence.

Yili Wu

yili_wu2004@yahoo.ca;

wuyili@wmu.edu.ca

†These authors have contributed equally to this work

Received: 08 September 2021

Accepted: 07 October 2021

Published: 08 November 2021

Citation:

Wang $Q$, Yao H, Liu W, Ya B,

Cheng $H$, Xing $Z$ and Wu Y (2021)

Microglia Polarization in Alzheimer's

Disease: Mechanisms and a Potential

Therapeutic Target.

Front. Aging Neurosci. 13:772717.

doi: 10.3389/fnagi.2021.772717

\section{Microglia Polarization in Alzheimer's Disease: Mechanisms and a Potential Therapeutic Target}

\author{
Qinqin Wang ${ }^{1 t}$, Hongmei Yao ${ }^{2 t}$, Wenyan Liu ${ }^{3}$, Bailiu Ya ${ }^{3}$, Hongju Cheng ${ }^{3}$, Zhenkai Xing ${ }^{1}$ \\ and Yili $W u^{4,5,6 *}$
}

${ }^{1}$ Shandong Collaborative Innovation Center for Diagnosis, Treatment and Behavioral Interventions of Mental Disorders, Institute of Mental Health, Jining Medical University, Jining, China, ${ }^{2}$ Affiliated Hospital of Jining Medical University, Jining, China, ${ }^{3}$ Department of Physiology, College of Basic Medicine, Jining Medical University, Jining, China, ${ }^{4}$ The Affiliated Kangning Hospital, Wenzhou Medical University, Wenzhou, China, ${ }^{5}$ Key Laboratory of Alzheimer's Disease of Zhejiang Province, Institute of Aging, School of Mental Health, Wenzhou Medical University, Wenzhou, China, ${ }^{6}$ Oujiang Laboratory, Wenzhou, China

Neuroinflammation regulated by microglia is one of the important factors involved in the pathogenesis of Alzheimer's disease (AD). Activated microglia exhibited phenotypes termed as M1 and M2 phenotypes separately. M1 microglia contribute to the development of inflammation via upregulating pro-inflammatory cytokines, while M2 microglia exert anti-inflammation effects through enhancing the expression of antiinflammation factors. Moreover, M1 and M2 microglia could be mutually transformed under various conditions. Both $\mathrm{M} 1$ and $\mathrm{M} 2$ microglia are implicated in $\mathrm{AD}$. Amyloid- $\beta(A \beta)$ and hyperphosphorylated tau are two major components of $A D$ pathological hallmarks, neuritic plaques, and neurofibrillary tangles. Both $A \beta$ and hyperphosphorylated tau were involved in microglial activation and subsequent inflammation, which further contribute to neuronal and synaptic loss in $\mathrm{AD}$. In this review, we summarized the roles of $\mathrm{M} 1$ and M2 microglia in $A D$ and underlying mechanisms, which will provide an insight into the role of microglia in the pathogenesis of $A D$ and highlight the therapeutic potential of modulating microglia.

Keywords: neuroinflammation, microglia activation, M1 microglia, M2 microglia, Alzheimer's disease

\section{INTRODUCTION}

As a devastating age-related brain disorder, Alzheimer's disease (AD) is characterized by progressive memory loss and cognitive deficits. Extracellular neuritic plaques, mainly consisting of amyloid- $\beta$ $(\mathrm{A} \beta)$ and intracellular neurofibrillary tangles (NFTs) containing hyperphosphorylated tau, are the major pathological hallmarks of AD (Palomer et al., 2019; Wang Z. et al., 2019; Frolich, 2020). Although genetic factors, abnormal cholesterol metabolism, and protein homeostasis deficiency have been reported to contribute to AD pathogenesis (Karch and Goate, 2015; Liebsch et al., 2019; van der Kant et al., 2019; Wang and Davis, 2021), molecular mechanisms of AD remain elusive.

Emerging evidence suggested that neuroinflammation mediated by microglia was an important feature of AD (Griciuc et al., 2019; Paouri and Georgopoulos, 2019). Microglia were activated through a classic pathway or an alternative pathway, termed M1 or M2 microglia (Wang Y. et al., 2019). M1 and M2 microglia had different properties and functions 
(Wang Y. et al., 2019), which were differentially involved in the pathogenesis of AD (Colton et al., 2006; Paouri and Georgopoulos, 2019). Advanced understanding of the roles of $\mathrm{M} 1$ and M2 microglia in $\mathrm{AD}$ will provide new clues about the pathological mechanisms and therapeutic targets for $\mathrm{AD}$. In this study, we summarized the recent progress about the functions of M1 and M2 microglia in $\mathrm{AD}$ pathogenesis and discussed underlying mechanisms.

\section{CHARACTERISTICS OF MICROGLIA}

\section{The Origin and Proliferation of Microglia}

Microglial cells play vital roles in regulating the homeostasis of the central nervous system (CNS; Subhramanyam et al., 2019). However, the origin of microglia is still controversial. Earlier studies indicated that microglial cells descended from meningeal macrophages, which were invaded into the brain during the late stage of embryonic development (Alliot et al., 1999). Later, it was found that microglia in the parenchyma of the CNS displayed macrophage markers and originated from the hemopoietic system (Perry et al., 1985; Lawson et al., 1990; Gordon et al., 1992; Alliot et al., 1999). Alliot et al. (1999) reported that microglia were derived from its progenitors originating from the hemopoietic cells as early as embryonic day 8 (E8) in the yolk sac. The microglial progenitors migrated into the brain subsequently and increased the number fast (Alliot et al., 1999). Ginhoux et al. (2010) showed that adult microglial cells were originated from the original myeloid progenitors before E8. Kierdorf et al. (2013) also reported that microglia were originated from the c-Kit positive erythro myeloid progenitors as early as E8 in the yolk sac. Early studies have demonstrated that there were three waves of hematopoiesis in the yolk sac during the embryonic development of mice (Yoder, 2014). Except for the classical microglial progenitors mainly produced during the first wave of hematopoiesis in the yolk sac, there also existed a microglial subpopulation originated from the second wave of hematopoiesis in the yolk sac (De et al., 2018). Taken together, most of these studies indicated that microglia were originated from the yolk sac during development. However, further investigation is necessary to clarify which wave of hematopoiesis and which type of cells in the yolk sac were mainly responsible for the microglial origination.

The microglia isolated from adult postmortem cerebral cortex exhibited proliferative ability in vitro and maintained its phenotype (Guo et al., 2016). The colony-stimulating factor-1 receptor (CSF1R) was vital for the development of microglia (Ginhoux et al., 2010; Erblich et al., 2011). Microglia could be repopulated after the withdrawal of CSF1R antagonism PLX5622 in both adult and aged mice (O'Neil et al., 2018). Moreover, CSF1R inhibitor PLX3397 treatment resulted in a dramatic decrease in microglia number, while microglia were restored rapidly after the removal of PLX3397 in mice (Elmore et al., 2014). Elmore et al. (2014) revealed that the rapidly repopulated microglia were from the nestin-positive nonmicroglial progenitor cells, indicating that the newborn microglia were derived from the nestin-positive progenitors but not the remaining microglia. However, Huang et al. (2018) found that the repopulated microglial cells were not derived from the blood cells or astrocytes, neurons, and NG2 cells but from CX3CR1positive microglia, demonstrating that the adult microglia could maintain its population through self-renewal. The inconformity of these two studies might result from the different protocols of drug treatment and different types of transgenic mice.

\section{Microglia Polarization}

Growing evidence indicated that two types of activated microglia existed, namely, M1 phenotype and M2 phenotype (Wang Y. et al., 2019), although there are different opinions about the existence of different phenotypes of microglia activation (Ransohoff, 2016). M1 phenotype microglia were the classical form of microglial activation and contributed to the development of inflammatory responses especially in neurodegeneration (Tang and Le, 2016; Kwon and Koh, 2020). M1 microglia could be induced by lipopolysaccharide (LPS)/interferon- $\gamma$ (IFN- $\gamma$; Tang and Le, 2016; Figure 1). M2 microglia were the alternative form of microglia activation and considered as the antiinflammatory response phenotype, contributing to tissue repair and neuroprotection (Figure 1). Moreover, M2 microglia could be induced by interleukin-4 (IL-4)/IL-13 (Tang and Le, 2016; Kwon and Koh, 2020; Figure 1). In addition, the expression levels of pro-inflammatory markers were enhanced significantly in M1 microglia, while the expression levels of anti-inflammatory factors increased in M2 microglia (Lam et al., 2017; Kwon and Koh, 2020). As the pro-inflammatory factors were significantly upregulated in M1 microglia, the inflammation-associated factors such as IL-1 $\beta$, IL-6, and inducible nitric oxide synthase (iNOS) were taken as the markers of M1 microglia (Jin et al., 2018; Figure 1). The expression of some relatively specific markers was upregulated in M2 microglia such as arginase 1 (ARG1), chitinase-3-like-3 (YM1, also called CHI3L3), and found in inflammatory zone 1 (FIZZ1; Jin et al., 2018; Veremeyko et al., 2018; Wu et al., 2019; Figure 1). Due to the diversity of diseases and different stages of diseases, it is essential to identify specific markers of microglial activation in various pathological conditions.

\section{Regulation of Microglia Polarization}

Accumulated evidence showed that microglial polarization could be regulated. For example, PGC-1-related coactivator (PRC) was significantly increased in M2 microglia induced by IL-4 (Mou et al., 2015). Consistently, the overexpression of PRC increased the mRNA expression of M2 microglial markers such as ARG1, FIZZ1, and YM1, promoting microglia activation toward the M2 phenotype (Figure 1; Mou et al., 2015). Trehalose$6,6^{\prime}$-dibehenate (TDB) could decrease the expression of M1 microglial markers such as the proIL- $1 \beta$ and IL- 6 induced by LPS (Mohanraj et al., 2019), while treatment with TDB also led to the significant increase of M2 microglia markers such as ARG1 and YM1/2 (Mohanraj et al., 2019; Figure 1). In addition, the dehydrocorydaline administration promoted the microglia toward the M2 phenotype in the spinal cord of a mouse model of bone cancer pain (Huo et al., 2018). Furthermore, TOPK, a mitogen-activated protein kinase, promoted the M2 


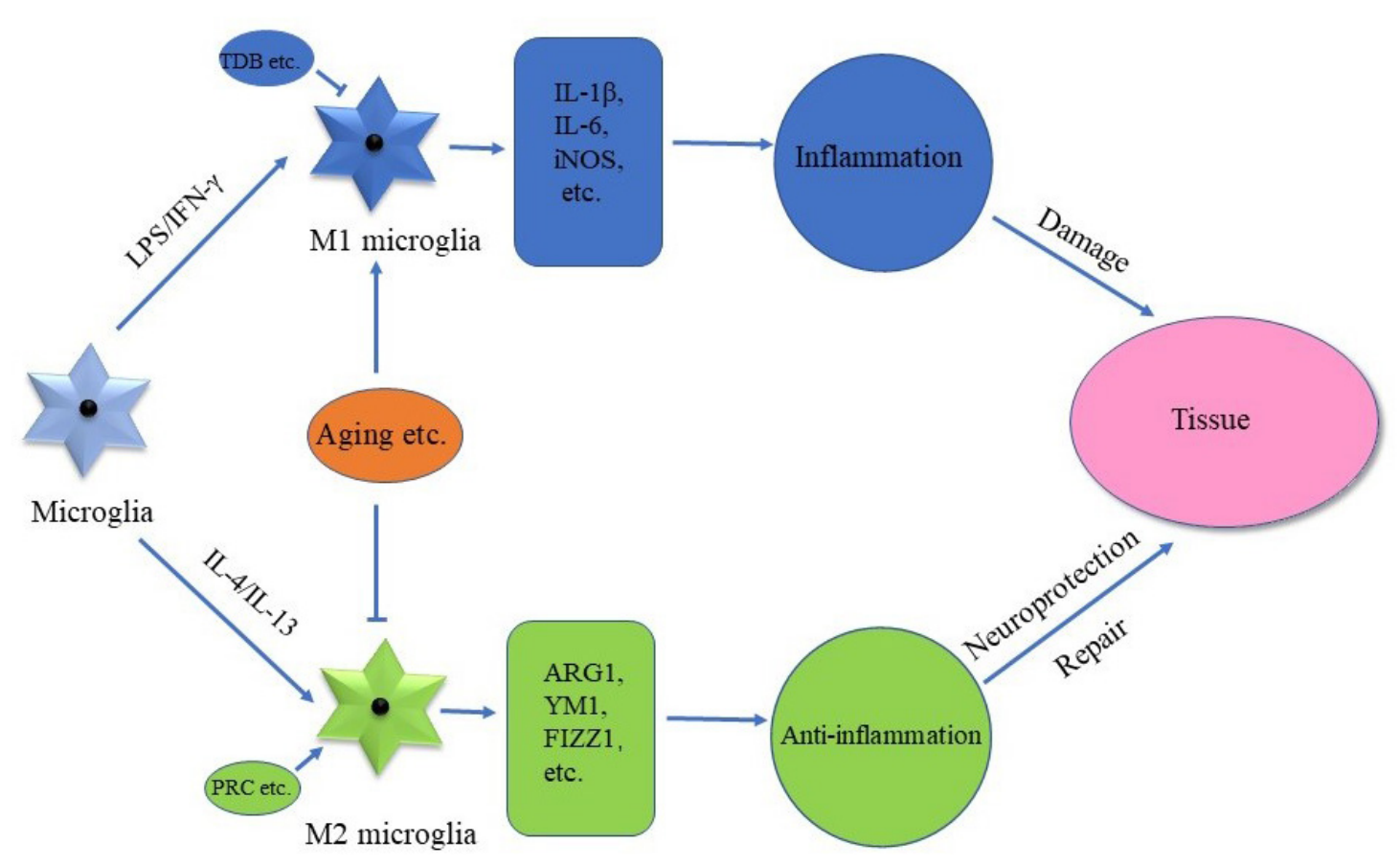

FIGURE 1 | A paradigm of M1 and M2 phenotypes of microglial cells. Microglia activation is categorized into two phenotypes, namely, M1 phenotype and M2 phenotype. M1 microglia could be induced by lipopolysaccharide/interferon- $\gamma$ (LPS/IFN- $\gamma$ ), leading to the increase of pro-inflammatory factors. M2 microglia could be induced by IL-4/L-13, which leads to the increase of anti-inflammatory factors. Thus, M1 microglia contribute to the inflammatory response, while M2 microglia contribute to the neuroprotection and repair processes of tissues.

microglial phenotype possibly through inhibiting the activity of histone deacetylases HDAC1 and HDAC2 (Han et al., 2018), while inhibiting $\mathrm{H} 3 \mathrm{~K} 27 \mathrm{me} 3$ demethylase Jmjd3 enhanced the pro-inflammatory responses and suppressed the microglial M2 phenotype (Tang et al., 2014). It indicated that microglial polarization could be regulated by many signaling pathways.

Importantly, evidence indicated that aging plays a pivotal role in the balance of M1 and M2 phenotypes. Aging promoted the M1 phenotype with higher levels of pro-inflammatory factors such as IL-1 $\beta$ and tumor necrosis factor- $\alpha$ (TNF- $\alpha$ ), while it decreased the activation of M2 microglia with a reduction of M2 markers such as ARG1, following peripheral surgery (Zhang Z. J. et al., 2019; Figure 1). When compared to 6-month-old mice, the expression of M1-related transcripts, such as S100A9 and CXCL13, tended to increase in 24-month-old mice treated with IL-1 $\beta$, IL-12, and TNF- $\alpha$, while an overall reduction of M2 microglia-associated markers such as ARG1, CHI3L3, and FIZZ1 was observed in 24-month-old mice treated with IL-4 and IL-13 cocktail. It indicated that aging facilitates the M1 phenotype but inhibits the M2 phenotype (Lee et al., 2013).

\section{DYSREGULATION OF MICROGLIA POLARIZATION IN ALZHEIMER'S DISEASE}

Increased mRNA of TNF- $\alpha$ was observed in the frontal lobe cortex of patients with AD (Colton et al., 2006). The mRNA of CHI3L1 and CHI3L2, which are the two YM1 closely related genes, was robustly increased in the cortex of the patients with AD (Colton et al., 2006). It indicated that both classically activated microglial cells and alternatively activated microglia existed in $\mathrm{AD}$ brains (Figure 2). However, cell population-specific alteration of these markers was undefined. For example, the expression of CD40 was upregulated in microglia of AD brains (Togo et al., 2000), while CD40 ligand deficiency resulted in a significant reduction of TNF$\alpha$ expression in cultured microglia of $\mathrm{AD}$ model mice (Tan et al., 1999). It indicated that the CD40-mediated pathway may play an important role in the M1 phenotype (Tan et al., 1999; Walker and Lue, 2015; Figure 2). Recent studies showed that activated microglia around amyloid plaques consisted of CD11c-positive and CD11c-negative subgroups in AD mice (Kamphuis et al., 2016). More interestingly, the transcriptional profiling analysis implied that CD11cpositive cells displayed increased immunosuppressive features counteracting the inflammatory response (Kamphuis et al., 2016). Hence, it was deduced that CD11c might be a marker of M2 microglia in AD (Figure 2).

Many susceptibility genes preferentially expressed in microglia of the aged human brain (e.g., TREM2, CD33, SORL1, and INPP5D) were associated with AD (Olah et al., 2018), while genetic analysis also indicated that various immunerelated genes were associated with $\mathrm{AD}$ risk (Karch and Goate, 2015). It is highly supported that microglia may play a key role in $\mathrm{AD}$ pathogenesis (Karch and Goate, 2015; 


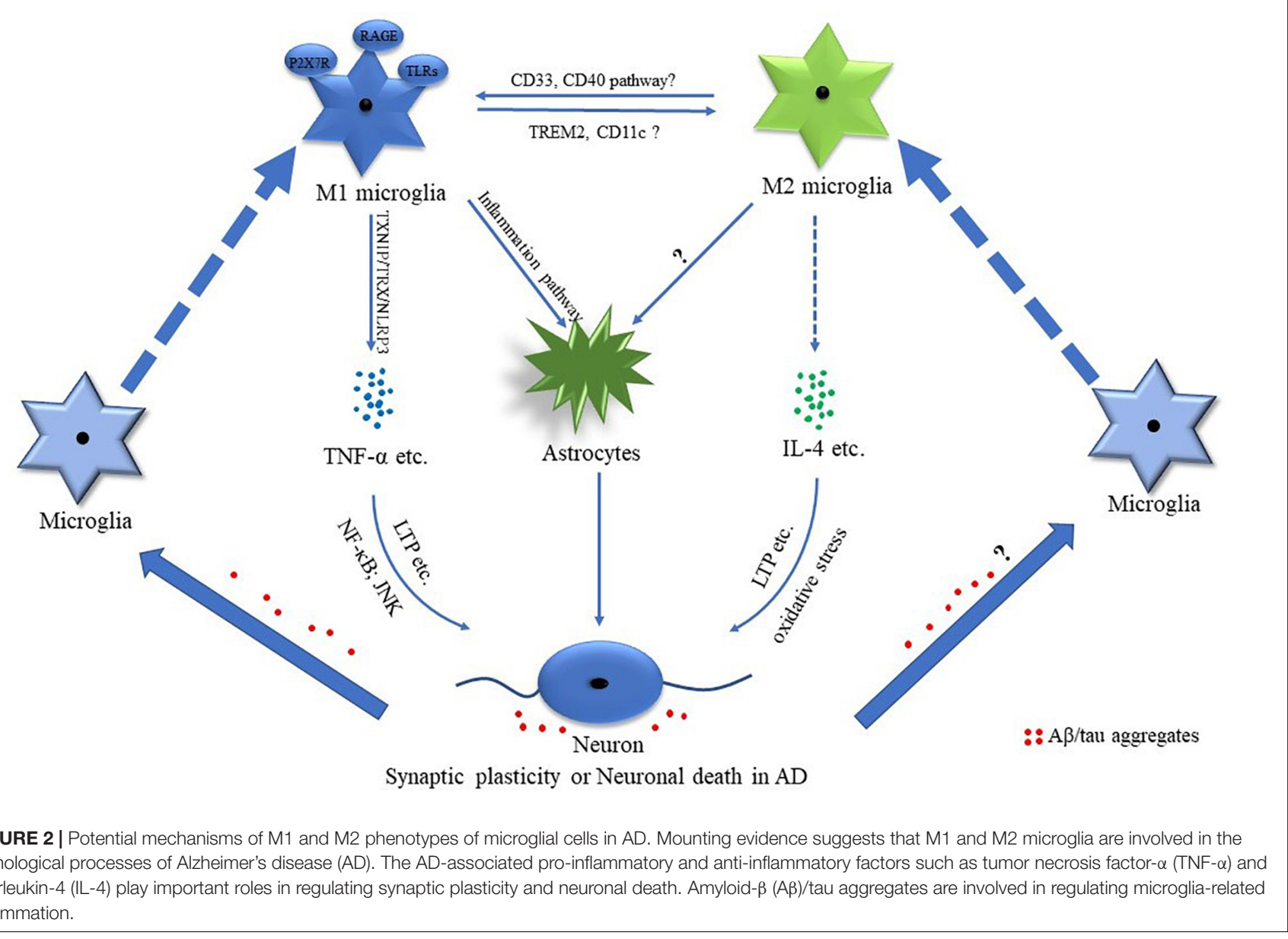

Katsumoto et al., 2018). For example, soluble triggering receptor expressed on myeloid cells 2 (sTREM2), a marker of microglia activation, was enhanced in cerebrospinal fluid at the stages of preclinical subjective cognitive decline, mild cognitive impairment (MCI), and $\mathrm{AD}$ dementia compared with healthy controls (Nordengen et al., 2019). In addition, TREM2 rare variants increased the risk of $\mathrm{AD}$ in Asian and European populations (Jonsson et al., 2013; Kleinberger et al., 2014; Jiang et al., 2016; Efthymiou and Goate, 2017). TREM2 mRNA was enhanced in patients with AD. Increased TREM2 promoted ARG1 expression and reduced the nitric oxide (NO) production (Zhang et al., 2018; Figure 2). In addition, TREM2 promoted microglial phagocytosis (Kawabori et al., 2015; Zhang et al., 2018). Moreover, TREM2 deficiency did accelerate AD progress (Wang et al., 2015; Ma et al., 2016; Efthymiou and Goate, 2017), which might be associated with TREM2 deficiency-mediated downregulation of ARG1 in microglial cells (Zhang et al., 2018).

CD33 was mainly expressed in microglia and macrophages in the brain (Zhao, 2019). It was reduced in peripheral mononuclear cells of patients with $\mathrm{AD}$, while it was increased in the frontal cortex of patients with $\mathrm{AD}$ (Griciuc et al., 2013; Hu et al., 2014). Consistently, the number of CD33positive microglia markedly increased in the cortex of $\mathrm{AD}$ cases
(Griciuc et al., 2013). CD33 knockout led to the increase of inflammasome genes and anti-inflammatory gene expression in AD mice (Griciuc et al., 2019), while a recent study further demonstrated that CD33 knockdown significantly decreased the pro-inflammatory-related transcripts in AD mice (Griciuc et al., 2020; Figure 2).

$\mathrm{P} 2 \mathrm{X} 7$ receptor $(\mathrm{P} 2 \mathrm{X} 7 \mathrm{R})$, which is a purinergic receptor, was significantly increased in microglial cells of the patients with $\mathrm{AD}$, which was associated with the pathology of $\mathrm{AD}$. The expression of $\mathrm{P} 2 \mathrm{X} 7 \mathrm{R}$ was higher in microglia of $\mathrm{AD}$ mice at both the advanced and the late stages, while no significant difference was detected at the early stage (Lee et al., 2011; Martinez-Frailes et al., 2019). The activation of P2X7R promoted microglia migration toward the senile plaques, while the inhibition of $\mathrm{P} 2 \mathrm{X} 7 \mathrm{R}$ promoted the phagocytosis of microglia (Martinez-Frailes et al., 2019). Moreover, P2X7R was involved in the degenerative neuron-induced microglial activation contributing to the activation of astrocytes resulting in the amplification of the neuroinflammation and neuronal damage (Yiangou et al., 2006; Glass et al., 2010). Consistently, P2X7R inhibition significantly reduced the expression of IL$1 \beta$ in spinal cord microglia of a rat pain model (Zhou et al., 2019). It indicated that P2X7R may be a key player of M1 microglia (Figure 2). 
Notably, microglia not only contributed to neuronal death by releasing pro-inflammatory cytokines but also exerted neuroprotective function via phagocytosis in AD (Pourbadie et al., 2018; Rangaraju et al., 2018). Specifically, the proportions of $\mathrm{CD}_{4} 4^{-} \mathrm{CXCR}^{+}{ }^{+}$and $\mathrm{CD} 44^{+} \mathrm{CXCR} 4^{-}$microglia were altered with age in $\mathrm{AD}$ mice. It suggested that aging may affect the profiles of M1 and M2 microglia in AD as CD44 and CXCR4 contributed to the pro-inflammatory and anti-inflammatory processes, respectively (Rangaraju et al., 2018). Moreover, it was indicated that M2 microglia switched into the M1 phenotype at the advanced stage of AD (Jimenez et al., 2008). However, the regulation of $\mathrm{M} 1 / \mathrm{M} 2$ microglia in $\mathrm{AD}$ remains elusive.

\section{THE ROLE OF MICROGLIA POLARIZATION IN THE PATHOGENESIS OF ALZHEIMER'S DISEASE}

\section{Mutual Regulation Between A $\beta$ Aggregates and Microglial Polarization}

Amyloid- $\beta$ was associated with the microglia activation in $\mathrm{AD}$ (Glass et al., 2010). The pathological analysis showed that activated microglia were accumulated around the plaques (Yin et al., 2017). Aggregated A $\beta$-induced M1 microglia were partially mediated by the receptor for advanced glycoxidation end-products (RAGE) and toll-like receptors (TLRs) (Figure 2), while the pro-inflammatory cytokines of M1 microglia further led to the activation of astrocytes, contributing to neuronal loss in AD (Glass et al., 2010; Desale and Chinnathambi, 2020; Figure 2). Early studies demonstrated that concentrated fibrillar $A \beta$ induced the microglia/monocyte activation with the increased expression of iNOS, which was TNF- $\alpha$ dependent, contributing to neuronal death (Combs et al., 2001; Chen et al., 2005). Recent studies showed that $\mathrm{A} \beta$-induced pro-inflammatory factors such as IL-6 and TNF- $\alpha$ were possibly medicated by thioredoxin-interacting protein (TXNIP)/thioredoxin (TRX)/NOD-like receptor pyrin domain-containing protein 3 (NLRP3) pathway in microglial cells (Feng and Zhang, 2019; Figure 2). Moreover, $A \beta$ could induce the activation of NLRP3 inflammasome in microglia, contributing to the activation of microglia toward M1 polarization (Halle et al., 2008; Liang et al., 2020; Zhang et al., 2020). Another study showed that $\mathrm{A} \beta$ led to the enhancement of pro-inflammatory cytokines such as IL-1 $\beta$ and IL-6 via regulating the homeostasis of ornithine decarboxylase and antizyme (Cheng et al., 2019). In addition, Cui et al. (2019) reported that enhanced NF-E2related factor 2 (Nrf2)/Kelch-like ECH-associated protein 1 (Keap1) signaling significantly attenuated the pro-inflammatory responses and oxidative stress induced by $A \beta$. These results indicated that $A \beta$ could directly induce or potentiate the M1 activation of microglia.

As the immune cells maintain the tissue homeostasis in the CNS, microglia cleared the debris and misfolded proteins by phagocytosis in the brain (Kettenmann et al., 2011; Zhou et al., 2020). It was reported that microglia cleared the $\mathrm{A} \beta$ via phagocytosis and proteases-mediated degradation
(Lopez-Valdes and Martinez-Coria, 2016). Chronically, the activation of M1 microglia released the cytotoxic factors and might have deficits in the clearance of $A \beta$ aggregates, accelerating the progress of AD (Glass et al., 2010; Lian et al., 2016; Zhang X. et al., 2019). The inactivation of CD33 promoted microglial uptake of $A \beta$, while the enhancement of $\mathrm{CD} 33$ significantly inhibited the uptake of $A \beta$ by microglia (Griciuc et al., 2013). $\mathrm{CD} 33$ promoted the development of $\mathrm{A} \beta$ aggregates, contributing to AD pathology (Griciuc et al., 2013). In addition, TLR2 deficiency led to the enhancement of $A \beta$ phagocytosis (Liu et al., 2012), while TLR2 knockout promoted M1 microglia to M2 microglia in bone marrow chimeric APP transgenic mice (Liu et al., 2012). Deferoxamine promoted the M2 activation of microglia and significantly reduced the $\mathrm{A} \beta$ deposits in $\mathrm{AD}$ mice (Zhang and He, 2017). Sarsasapogenin-AA13 significantly alleviated $A \beta$-induced cognitive deficiency possibly through reducing the M1 activation, increasing the M2 activation, and enhancing $A \beta$ clearance in mice (Huang et al., 2017). It was suggested that treadmill exercise enhanced the M2 activation and decreased the M1 activation of microglia, contributing to the decrease of $\mathrm{A} \beta$ deposition and improvement of cognition in AD mice (Zhang X. et al., 2019). These results indicated that microglial polarization has a significant effect on $A \beta$ deposition, i.e., M2 microglia might have a strong ability to clear $\mathrm{A} \beta$. However, further investigation is needed to elucidate the association between microglial activation and $\mathrm{A} \beta$ clearance.

\section{Mutual Regulation Between Tau Aggregates and Microglial Polarization}

Increasing evidence indicated that tau was closely associated with microglia activation. The tau-induced activation of microglia was medicated by the NLRP3 inflammasome, contributing to the release of IL-1 $\beta$ (Ising et al., 2019). NLRP3 knockout greatly inhibited tau hyperphosphorylation induced by brain homogenates of APP/PS1 mice, indicating the vital roles of NLRP3 in tau pathology (Ising et al., 2019). Ionized calcium-binding adaptor molecule-1 (Iba1) was often taken as the marker of microglia activation following inflammatory stimulation (Hoogland et al., 2015). Tau aggregates could spread the localization of Ibal to the cell membrane, and tau oligomer also induced the increase of cytosolic Ibal levels in microglia, indicating that tau could drive the pro-inflammatory activation of microglia in $\mathrm{AD}$ (Das et al., 2020; Figure 2). On the other hand, the proinflammatory cytokines released from the activated microglia also contributed to the regulation of tau phosphorylation and the formation of tau aggregates (Li et al., 2003; Barron et al., 2017; Leyns and Holtzman, 2017). For example, IL-1 $\beta$ secreted by microglia triggered tau phosphorylation through the p38 mitogen-activated protein kinase (MAPK) pathway (Li et al., 2003; Barron et al., 2017; Desale and Chinnathambi, 2020). TNF- $\alpha$ induced the hyperphosphorylation of tau while the long-term treatment of TNF- $\alpha$ led to a marked decrease of tau hyperphosphorylation in $\mathrm{AD}$ mice (Janelsins et al., 2008). However, the immunohistochemical analysis showed that another pro-inflammatory factor IFN- $\gamma$ significantly 
decreased the levels of hyperphosphorylated tau in the brain of $\mathrm{AD}$ mice, indicating the potential roles of IFN- $\gamma$ in tau hyperphosphorylation (Mastrangelo et al., 2009). Moreover, it was suggested that microglia exerted the cleaning function to clear tau oligomers by phagocytosis in AD (Das et al., 2020). It indicated that tau aggregates and M1 microglial activation are possibly mutually regulated.

\section{Synaptic Dysfunction and Neuronal Death Mediated by Microglia Polarization}

Emerging evidence demonstrated that the AD-associated proinflammatory factors such as IL- $1 \beta$ and TNF- $\alpha$ played important roles in synaptic dysfunction and neuronal death (Gaur and Agnihotri, 2015; Pettigrew et al., 2016; Rincon-Lopez et al., 2017; Rizzo et al., 2018; Xiao et al., 2020). For example, increased IL-1 $\beta$ was observed during the long-term potentiation (LTP), while IL-1 receptor blockade led to the impairment of LTP in the hippocampus, indicating that IL- $1 \beta$ is implicated in the regulation of synaptic plasticity (Schneider et al., 1998; Coogan et al., 1999; Avital et al., 2003). Moreover, IL-1 $\beta$ promoted neuronal death in the hippocampus of developing rats with status epilepticus (Rincon-Lopez et al., 2017). In addition, TNF$\alpha$ mediated polyinosinic-polycytidylic acid [Poly(I:C)]-induced elimination and formation of the dendritic spine in wild-type mice (Garre et al., 2017). Consistently, dominant-negative TNF- $\alpha$ significantly inhibited dendritic spine elimination and formation in the somatosensory cortex of experimental autoimmune encephalomyelitis (EAE) mice (Yang et al., 2013). TNF- $\alpha$ significantly increased the LTP levels of the hippocampus (Pettigrew et al., 2016; Figure 2). Furthermore, TNF- $\alpha$ induced neuronal apoptosis possibly through the upregulation of c-Jun $\mathrm{N}$-terminal kinase (JNK) activation and increased nuclear factor kappa B (NF-кB) p65 and iNOS (Yang et al., 2002; Kraft et al., 2009; Xiao et al., 2020). The aforementioned evidence indicated the important role of TNF- $\alpha$ in the regulation of synaptic plasticity and neuronal death (Figure 2).

Anti-inflammatory cytokines were implicated in synaptic plasticity and neuronal death. A significant increase of IL-4 was observed in patients with AD (King et al., 2018), which played important roles in the protection of synaptic plasticity and neuronal death (Maher et al., 2005; Bhattarai et al., 2016; Hernandez-Espinosa et al., 2019; Jeong et al., 2019; Taipa et al., 2019). Maher et al. (2005) reported that the decrease of IL-4-related signaling contributed to the LTP deficits in the aged rat (Figure 2), while IL-4 and IL-10 administration significantly decreased the neuronal injury induced by excitotoxic damage in wild-type animals (Hernandez-Espinosa et al., 2019). However, IL-4 aggravated the neuronal death induced by prothrombin kringle-2 ( $\mathrm{pKr}-2)$ possibly through regulating the oxidative stress (Jeong et al., 2019; Figure 2). All these results implicated that the AD-related pro-inflammatory and antiinflammatory cytokines were involved in modulating synaptic plasticity and neuronal death.

Taken together, besides involving in the regulation of the synaptic dysfunction and neuronal death by the inflammatoryrelated factors, the activated microglia could also affect the pathological process of $\mathrm{AD}$ through regulating $\mathrm{A} \beta$ and tau deposition. It is noteworthy that different pro-inflammatory cytokines or anti-inflammatory factors might have their unique function in $\mathrm{A} \beta$ and tau pathology in $\mathrm{AD}$.

\section{THE THERAPEUTIC POTENTIAL OF TARGETING MICROGLIA POLARIZATION}

The $A \beta$ and tau aggregates mainly induced M1 microglia (Glass et al., 2010; Das et al., 2020), while the inflammation mediated by microglia was involved in the formation of $A \beta$ and tau aggregates, as well as the synaptic and neuronal loss contributing to the neurodegeneration (Jimenez et al., 2008; Glass et al., 2010; Gaur and Agnihotri, 2015; Lian et al., 2016; Pettigrew et al., 2016; Rincon-Lopez et al., 2017; Rizzo et al., 2018; Zhang X. et al., 2019; Xiao et al., 2020). Thus, it was crucial to clarify the molecular mechanisms of microglial activation in $\mathrm{AD}$, which will help to find the efficient drugs that targeted the microglial activation such as the inhibition of pro-inflammation or the promotion of anti-inflammation. For example, CD33 inactivation promoted the uptake of $\mathrm{A} \beta$ by microglia (Griciuc et al., 2013). Consistently, CD33 knockdown significantly decreased the proinflammatory-related transcripts and $\mathrm{A} \beta$ plaque in $\mathrm{AD}$ mice at an early age (Griciuc et al., 2020). Thus, CD33 might be a potential target for $\mathrm{AD}$ treatment. In addition, P2X7R was indicated as a key player in M1 microglia (Zhou et al., 2019). Targeting P2X7R might be beneficial for $\mathrm{AD}$ treatment by inhibiting M1 microglia. Moreover, increased TREM2 promoted M2 phenotype, indicating that increasing the TREM2 activity may be a potential approach for $\mathrm{AD}$ treatment by promoting M2 microglia (Zhang et al., 2018). Furthermore, TLR2 knockout contributed to the transition from M1 to M2 microglia (Liu et al., 2012). It suggested that targeting TLR2 might be a potential approach for $\mathrm{AD}$ treatment by promoting M1 to M2 shift. Therefore, efficiently targeting the abovementioned or more candidates facilitating the shift from M1 to M2 may have therapeutic potential for AD.

\section{CONCLUSION}

Mounting evidence has shown that the microglia-associated neuroinflammation was one of the major hallmarks of $\mathrm{AD}$, while the microglial activation was correlated with the progress of AD. M1 and M2 microglia, i.e., pro-inflammation and anti-inflammation phenotypes, played differential roles in $\mathrm{AD}$ pathogenesis although precise characteristics and regulation of them still need to be fully elucidated. Balancing M1 and M2 microglia or promoting the shift from M1 to M2 might have therapeutic potential for $\mathrm{AD}$ treatment.

\section{AUTHOR CONTRIBUTIONS}

QW and HY wrote the manuscript. WL, BY, HC, and ZX contributed to the revision of the manuscript. YW conducted the editing of the manuscript. All authors approved the final manuscript. 


\section{FUNDING}

This study was financially supported by the NSFC cultivation project of Jining Medical University (JYP2019KJ20), Technology Development Project of Shandong Province Medicine and Health Science (No. 202002081082), Scientific and Technological Development Program of the Health and Family Planning Commission of Shandong Province [201407 (No. 7 in 2014)],

\section{REFERENCES}

Alliot, F., Godin, I., and Pessac, B. (1999). Microglia derive from progenitors, originating from the yolk sac, and which proliferate in the brain. Brain Res. Dev. Brain Res. 117, 145-152.

Avital, A., Goshen, I., Kamsler, A., Segal, M., Iverfeldt, K., Richter-Levin, G., et al. (2003). Impaired interleukin-1 signaling is associated with deficits in hippocampal memory processes and neural plasticity. Hippocampus 13, 826834. doi: 10.1002/hipo.10135

Barron, M., Gartlon, J., Dawson, L. A., Atkinson, P. J., and Pardon, M. C. (2017). A state of delirium: deciphering the effect of inflammation on tau pathology in Alzheimer's disease. Exp. Gerontol. 94, 103-107. doi: 10.1016/j.exger.2016.12. 006

Bhattarai, P., Thomas, A. K., Cosacak, M. I., Papadimitriou, C., Mashkaryan, V., Froc, C., et al. (2016). IL4/STAT6 Signaling Activates Neural Stem Cell Proliferation and Neurogenesis upon Amyloid-beta42 Aggregation in Adult Zebrafish Brain. Cell Rep. 17, 941-948. doi: 10.1016/j.celrep.2016.09.075

Chen, J., Zhou, Y., Mueller-Steiner, S., Chen, L. F., Kwon, H., Yi, S., et al. (2005). SIRT1 protects against microglia-dependent amyloid-beta toxicity through inhibiting NF-kappaB signaling. J. Biol. Chem. 280, 40364-40374. doi: 10.1074/ jbc.M509329200

Cheng, Y. W., Chang, C. C., Chang, T. S., Li, H. H., Hung, H. C., Liu, G. Y., et al. (2019). Abeta stimulates microglial activation through antizyme-dependent downregulation of ornithine decarboxylase. J. Cell. Physiol. 234, 9733-9745. doi: $10.1002 /$ jcp. 27659

Colton, C. A., Mott, R. T., Sharpe, H., Xu, Q., Van Nostrand, W. E., and Vitek, M. P. (2006). Expression profiles for macrophage alternative activation genes in AD and in mouse models of AD. J. Neuroinflammation 3:27. doi: 10.1186/17422094-3-27

Combs, C. K., Karlo, J. C., Kao, S. C., and Landreth, G. E. (2001). betaAmyloid stimulation of microglia and monocytes results in TNFalphadependent expression of inducible nitric oxide synthase and neuronal apoptosis. J. Neurosci. 21, 1179-1188. doi: 10.1523/JNEUROSCI.21-04-01179. 2001

Coogan, A. N., O’Neill, L. A., and O'Connor, J. J. (1999). The P38 mitogenactivated protein kinase inhibitor SB203580 antagonizes the inhibitory effects of interleukin-1beta on long-term potentiation in the rat dentate gyrus in vitro. Neuroscience 93, 57-69. doi: 10.1016/s0306-4522(99)00100-1

Cui, B., Zhang, S., Wang, Y., and Guo, Y. (2019). Farrerol attenuates beta-amyloidinduced oxidative stress and inflammation through Nrf2/Keap1 pathway in a microglia cell line. Biomed. Pharmacother. 109, 112-119. doi: 10.1016/j.biopha. 2018.10.053

Das, R., Balmik, A. A., and Chinnathambi, S. (2020). Phagocytosis of full-length Tau oligomers by Actin-remodeling of activated microglia. J. Neuroinflammation 17:10. doi: 10.1186/s12974-019-1694-y

De, S., Van Deren, D., Peden, E., Hockin, M., Boulet, A., Titen, S., et al. (2018). Two distinct ontogenies confer heterogeneity to mouse brain microglia. Development 145:dev175901. doi: 10.1242/dev.152306

Desale, S. E., and Chinnathambi, S. (2020). Role of dietary fatty acids in microglial polarization in Alzheimer's disease. J. Neuroinflammation 17:93. doi: 10.1186/ s12974-020-01742-3

Efthymiou, A. G., and Goate, A. M. (2017). Late onset Alzheimer's disease genetics implicates microglial pathways in disease risk. Mol. Neurodegener. 12:43. doi: 10.1186/s13024-017-0184-x

Elmore, M. R., Najafi, A. R., Koike, M. A., Dagher, N. N., Spangenberg, E. E., Rice, R. A., et al. (2014). Colony-stimulating factor 1 receptor signaling is necessary
Research Fund for the Lin He's Academician Workstation of New Medicine and Clinical Translation in Jining Medical University (JYHL2018MS03), A Project of Shandong Province Higher Educational Science and Technology Program (J18KA124), A Project of Shandong Province Higher Educational Science and Technology Program (J18KB119), National Natural Science Foundation of China (No. 81703490), and Natural Science Foundation of Shandong Province (No. ZR2015HQ019).

for microglia viability, unmasking a microglia progenitor cell in the adult brain. Neuron 82, 380-397. doi: 10.1016/j.neuron.2014.02.040

Erblich, B., Zhu, L., Etgen, A. M., Dobrenis, K., and Pollard, J. W. (2011). Absence of colony stimulation factor-1 receptor results in loss of microglia, disrupted brain development and olfactory deficits. PLoS One 6:e26317. doi: 10.1371/ journal.pone.0026317

Feng, L., and Zhang, L. (2019). Resveratrol Suppresses Abeta-Induced Microglial Activation Through the TXNIP/TRX/NLRP3 Signaling Pathway. DNA Cell Biol. 38, 874-879. doi: 10.1089/dna.2018.4308

Frolich, L. (2020). Alzheimer's disease - the 'microbial hypothesis' from a clinical and neuroimaging perspective. Psychiatry Res. Neuroimaging 306:111181. doi: 10.1016/j.pscychresns.2020.111181

Garre, J. M., Silva, H. M., Lafaille, J. J., and Yang, G. (2017). CX3CR1(+) monocytes modulate learning and learning-dependent dendritic spine remodeling via TNF-alpha. Nat. Med. 23, 714-722. doi: 10.1038/nm.4340

Gaur, S., and Agnihotri, R. (2015). Alzheimer's disease and chronic periodontitis: is there an association? Geriatr. Gerontol. Int. 15, 391-404. doi: 10.1111/ggi.12425

Ginhoux, F., Greter, M., Leboeuf, M., Nandi, S., See, P., Gokhan, S., et al. (2010). Fate mapping analysis reveals that adult microglia derive from primitive macrophages. Science 330, 841-845. doi: 10.1126/science.1194637

Glass, C. K., Saijo, K., Winner, B., Marchetto, M. C., and Gage, F. H. (2010). Mechanisms underlying inflammation in neurodegeneration. Cell 140, 918934. doi: 10.1016/j.cell.2010.02.016

Gordon, S., Lawson, L., Rabinowitz, S., Crocker, P. R., Morris, L., and Perry, V. H. (1992). Antigen markers of macrophage differentiation in murine tissues. Curr. Top. Microbiol. Immunol. 181, 1-37.

Griciuc, A., Federico, A. N., Natasan, J., Forte, A. M., McGinty, D., Nguyen, H., et al. (2020). Gene therapy for Alzheimer's disease targeting CD33 reduces amyloid beta accumulation and neuroinflammation. Hum. Mol. Genet. 29, 2920-2935. doi: 10.1093/hmg/ddaa179

Griciuc, A., Patel, S., Federico, A. N., Choi, S. H., Innes, B. J., Oram, M. K., et al. (2019). TREM2 Acts Downstream of CD33 in Modulating Microglial Pathology in Alzheimer's Disease. Neuron 103, 820-835.e7. doi: 10.1016/j.neuron.2019.06. 010

Griciuc, A., Serrano-Pozo, A., Parrado, A. R., Lesinski, A. N., Asselin, C. N., Mullin, K., et al. (2013). Alzheimer's disease risk gene CD33 inhibits microglial uptake of amyloid beta. Neuron 78, 631-643. doi: 10.1016/j.neuron.2013.04.014

Guo, L., Rezvanian, A., Kukreja, L., Hoveydai, R., Bigio, E. H., Mesulam, M. M., et al. (2016). Postmortem Adult Human Microglia Proliferate in Culture to High Passage and Maintain Their Response to Amyloid-beta. J. Alzheimers Dis. 54, 1157-1167. doi: 10.3233/JAD-160394

Halle, A., Hornung, V., Petzold, G. C., Stewart, C. R., Monks, B. G., Reinheckel, T., et al. (2008). The NALP3 inflammasome is involved in the innate immune response to amyloid-beta. Nat. Immunol. 9, 857-865. doi: 10.1038/ni.1636

Han, Z., Zhao, H., Tao, Z., Wang, R., Fan, Z., Luo, Y., et al. (2018). TOPK Promotes Microglia/Macrophage Polarization towards M2 Phenotype via Inhibition of HDAC1 and HDAC2 Activity after Transient Cerebral Ischemia. Aging Dis. 9, 235-248. doi: 10.14336/AD.2017.0328

Hernandez-Espinosa, D. R., Massieu, L., Montiel, T., and Moran, J. (2019). Role of NADPH oxidase- 2 in the progression of the inflammatory response secondary to striatum excitotoxic damage. J. Neuroinflammation 16:91. doi: 10.1186/ s12974-019-1478-4

Hoogland, I. C., Houbolt, C., van Westerloo, D. J., van Gool, W. A., and van de Beek, D. (2015). Systemic inflammation and microglial activation: systematic review of animal experiments. J. Neuroinflammation 12:114. doi: 10.1186/ s12974-015-0332-6 
Hu, N., Tan, M. S., Sun, L., Jiang, T., Wang, Y. L., Tan, L., et al. (2014). Decreased expression of CD33 in peripheral mononuclear cells of Alzheimer's disease patients. Neurosci. Lett. 563, 51-54. doi: 10.1016/j.neulet.2014.01.004

Huang, C., Dong, D., Jiao, Q., Pan, H., Ma, L., and Wang, R. (2017). Sarsasapogenin-AA13 ameliorates Abeta-induced cognitive deficits via improving neuroglial capacity on Abeta clearance and antiinflammation. CNS Neurosci. Ther. 23, 498-509. doi: 10.1111/cns.12697

Huang, Y., Xu, Z., Xiong, S., Sun, F., Qin, G., Hu, G., et al. (2018). Repopulated microglia are solely derived from the proliferation of residual microglia after acute depletion. Nat. Neurosci. 21, 530-540. doi: 10.1038/s41593-018-0090-8

Huo, W., Zhang, Y., Liu, Y., Lei, Y., Sun, R., Zhang, W., et al. (2018). Dehydrocorydaline attenuates bone cancer pain by shifting microglial M1/M2 polarization toward the M2 phenotype. Mol. Pain 14:1744806918781733. doi: $10.1177 / 1744806918781733$

Ising, C., Venegas, C., Zhang, S., Scheiblich, H., Schmidt, S. V., Vieira-Saecker, A., et al. (2019). NLRP3 inflammasome activation drives tau pathology. Nature 575, 669-673. doi: 10.1038/s41586-019-1769-z

Janelsins, M. C., Mastrangelo, M. A., Park, K. M., Sudol, K. L., Narrow, W. C., Oddo, S., et al. (2008). Chronic neuron-specific tumor necrosis factor-alpha expression enhances the local inflammatory environment ultimately leading to neuronal death in 3xTg-AD mice. Am. J. Pathol. 173, 1768-1782. doi: 10.2353/ajpath.2008.080528

Jeong, J. Y., Chung, Y. C., and Jin, B. K. (2019). Interleukin-4 and Interleukin13 Exacerbate Neurotoxicity of Prothrombin Kringle-2 in Cortex In Vivo via Oxidative Stress. Int. J. Mol. Sci. 20:1927. doi: 10.3390/ijms2008 1927

Jiang, T., Tan, L., Chen, Q., Tan, M. S., Zhou, J. S., Zhu, X. C., et al. (2016). A rare coding variant in TREM2 increases risk for Alzheimer's disease in Han Chinese. Neurobiol. Aging 42, 217.e1-3. doi: 10.1016/j.neurobiolaging.2016.02.023

Jimenez, S., Baglietto-Vargas, D., Caballero, C., Moreno-Gonzalez, I., Torres, M., Sanchez-Varo, R., et al. (2008). Inflammatory response in the hippocampus of PS1M146L/APP751SL mouse model of Alzheimer's disease: age-dependent switch in the microglial phenotype from alternative to classic. J. Neurosci. 28, 11650-11661. doi: 10.1523/JNEUROSCI.3024-08.2008

Jin, M. M., Wang, F., Qi, D., Liu, W. W., Gu, C., Mao, C. J., et al. (2018). A Critical Role of Autophagy in Regulating Microglia Polarization in Neurodegeneration. Front. Aging Neurosci. 10:378. doi: 10.3389/fnagi.2018.00378

Jonsson, T., Stefansson, H., Steinberg, S., Jonsdottir, I., Jonsson, P. V., Snaedal, J., et al. (2013). Variant of TREM2 associated with the risk of Alzheimer's disease. N. Engl. J. Med. 368, 107-116. doi: 10.1056/NEJMoa1211103

Kamphuis, W., Kooijman, L., Schetters, S., Orre, M., and Hol, E. M. (2016). Transcriptional profiling of CD11c-positive microglia accumulating around amyloid plaques in a mouse model for Alzheimer's disease. Biochim. Biophys. Acta 1862, 1847-1860. doi: 10.1016/j.bbadis.2016.07.007

Karch, C. M., and Goate, A. M. (2015). Alzheimer's disease risk genes and mechanisms of disease pathogenesis. Biol. Psychiatry 77, 43-51. doi: 10.1016/j. biopsych.2014.05.006

Katsumoto, A., Takeuchi, H., Takahashi, K., and Tanaka, F. (2018). Microglia in Alzheimer's Disease: risk Factors and Inflammation. Front. Neurol. 9:978. doi: 10.3389/fneur.2018.00978

Kawabori, M., Kacimi, R., Kauppinen, T., Calosing, C., Kim, J. Y., Hsieh, C. L., et al. (2015). Triggering receptor expressed on myeloid cells 2 (TREM2) deficiency attenuates phagocytic activities of microglia and exacerbates ischemic damage in experimental stroke. J. Neurosci. 35, 3384-3396. doi: 10.1523/JNEUROSCI. 2620-14.2015

Kettenmann, H., Hanisch, U. K., Noda, M., and Verkhratsky, A. (2011). Physiology of microglia. Physiol. Rev. 91, 461-553. doi: 10.1152/physrev.00011.2010

Kierdorf, K., Erny, D., Goldmann, T., Sander, V., Schulz, C., Perdiguero, E. G., et al. (2013). Microglia emerge from erythromyeloid precursors via Pu.1- and Irf8-dependent pathways. Nat. Neurosci. 16, 273-280. doi: 10.1038/nn.3318

King, E., O’Brien, J. T., Donaghy, P., Morris, C., Barnett, N., Olsen, K., et al. (2018). Peripheral inflammation in prodromal Alzheimer's and Lewy body dementias. J. Neurol. Neurosurg. Psychiatry 89, 339-345. doi: 10.1136/jnnp-2017-317134

Kleinberger, G., Yamanishi, Y., Suarez-Calvet, M., Czirr, E., Lohmann, E., Cuyvers, E., et al. (2014). TREM2 mutations implicated in neurodegeneration impair cell surface transport and phagocytosis. Sci. Transl. Med. 6:243ra286. doi: 10.1126/scitranslmed.3009093
Kraft, A. D., McPherson, C. A., and Harry, G. J. (2009). Heterogeneity of microglia and TNF signaling as determinants for neuronal death or survival. Neurotoxicology 30, 785-793. doi: 10.1016/j.neuro.2009.07.001

Kwon, H. S., and Koh, S. H. (2020). Neuroinflammation in neurodegenerative disorders: the roles of microglia and astrocytes. Transl. Neurodegener. 9:42. doi: 10.1186/s40035-020-00221-2

Lam, D., Lively, S., and Schlichter, L. C. (2017). Responses of rat and mouse primary microglia to pro- and anti-inflammatory stimuli: molecular profiles, $\mathrm{K}(+)$ channels and migration. J. Neuroinflammation 14:166. doi: 10.1186/ s12974-017-0941-3

Lawson, L. J., Perry, V. H., Dri, P., and Gordon, S. (1990). Heterogeneity in the distribution and morphology of microglia in the normal adult mouse brain. Neuroscience 39, 151-170. doi: 10.1016/0306-4522(90)90229-w

Lee, D. C., Ruiz, C. R., Lebson, L., Selenica, M. L., Rizer, J., Hunt, J. B. Jr., et al. (2013). Aging enhances classical activation but mitigates alternative activation in the central nervous system. Neurobiol. Aging 34, 1610-1620. doi: 10.1016/j. neurobiolaging.2012.12.014

Lee, H. G., Won, S. M., Gwag, B. J., and Lee, Y. B. (2011). Microglial P2X(7) receptor expression is accompanied by neuronal damage in the cerebral cortex of the APPswe/PS1dE9 mouse model of Alzheimer's disease. Exp. Mol. Med. 43, 7-14. doi: 10.3858/emm.2011.43.1.001

Leyns, C. E. G., and Holtzman, D. M. (2017). Glial contributions to neurodegeneration in tauopathies. Mol. Neurodegener. 12:50. doi: 10.1186/ s13024-017-0192-x

Li, Y., Liu, L., Barger, S. W., and Griffin, W. S. (2003). Interleukin-1 mediates pathological effects of microglia on tau phosphorylation and on synaptophysin synthesis in cortical neurons through a p38-MAPK pathway. J. Neurosci. 23, 1605-1611. doi: 10.1523/JNEUROSCI.23-05-01605.2003

Lian, H., Litvinchuk, A., Chiang, A. C., Aithmitti, N., Jankowsky, J. L., and Zheng, H. (2016). Astrocyte-Microglia Cross Talk through Complement Activation Modulates Amyloid Pathology in Mouse Models of Alzheimer's Disease. J. Neurosci. 36, 577-589. doi: 10.1523/JNEUROSCI.2117-15.2016

Liang, F., Huang, T., Li, B., Zhao, Y., Zhang, X., and Xu, B. (2020). Highintensity interval training and moderate-intensity continuous training alleviate beta-amyloid deposition by inhibiting NLRP3 inflammasome activation in APPswe/PS1dE9 mice. Neuroreport 31, 425-432. doi: 10.1097/WNR. 0000000000001429

Liebsch, F., Kulic, L., Teunissen, C., Shobo, A., Ulku, I., Engelschalt, V., et al. (2019). Abeta34 is a BACE1-derived degradation intermediate associated with amyloid clearance and Alzheimer's disease progression. Nat. Commun. 10:2240. doi: 10.1038/s41467-019-10152-w

Liu, S., Liu, Y., Hao, W., Wolf, L., Kiliaan, A. J., Penke, B., et al. (2012). TLR2 is a primary receptor for Alzheimer's amyloid beta peptide to trigger neuroinflammatory activation. J. Immunol. 188, 1098-1107. doi: 10.4049/ jimmunol.1101121

Lopez-Valdes, H. E., and Martinez-Coria, H. (2016). The Role of Neuroinflammation in Age-Related Dementias. Rev. Invest. Clin. 68, 40-48.

Ma, L., Allen, M., Sakae, N., Ertekin-Taner, N., Graff-Radford, N. R., Dickson, D. W., et al. (2016). Expression and processing analyses of wild type and p.R47H TREM2 variant in Alzheimer's disease brains. Mol. Neurodegener. 11:72. doi: 10.1186/s13024-016-0137-9

Maher, F. O., Nolan, Y., and Lynch, M. A. (2005). Downregulation of IL-4induced signalling in hippocampus contributes to deficits in LTP in the aged rat. Neurobiol. Aging 26, 717-728. doi: 10.1016/j.neurobiolaging.2004.07.002

Martinez-Frailes, C., Di Lauro, C., Bianchi, C., de Diego-Garcia, L., Sebastian-Serrano, A., Bosca, L., et al. (2019). Amyloid Peptide Induced Neuroinflammation Increases the P2X7 Receptor Expression in Microglial Cells, Impacting on Its Functionality. Front. Cell. Neurosci. 13:143. doi: $10.3389 /$ fncel.2019.00143

Mastrangelo, M. A., Sudol, K. L., Narrow, W. C., and Bowers, W. J. (2009). Interferon-\{gamma\} differentially affects Alzheimer's disease pathologies and induces neurogenesis in triple transgenic-AD mice. Am. J. Pathol. 175, 20762088. doi: 10.2353/ajpath.2009.090059

Mohanraj, M., Sekar, P., Liou, H. H., Chang, S. F., and Lin, W. W. (2019). The Mycobacterial Adjuvant Analogue TDB Attenuates Neuroinflammation via Mincle-Independent PLC-gamma1/PKC/ERK Signaling and Microglial Polarization. Mol. Neurobiol. 56, 1167-1187. doi: 10.1007/s12035-018-1135-4 
Mou, C., Liu, B., Wang, M., Jiang, M., and Han, T. (2015). PGC-1-related coactivator (PRC) is an important regulator of microglia M2 polarization. J. Mol. Neurosci. 55, 69-75. doi: 10.1007/s12031-014-0315-6

Nordengen, K., Kirsebom, B. E., Henjum, K., Selnes, P., Gisladottir, B., Wettergreen, M., et al. (2019). Glial activation and inflammation along the Alzheimer's disease continuum. J. Neuroinflammation 16:46. doi: 10.1186/ s12974-019-1399-2

Olah, M., Patrick, E., Villani, A. C., Xu, J., White, C. C., Ryan, K. J., et al. (2018). A transcriptomic atlas of aged human microglia. Nat. Commun. 9:539. doi: 10.1038/s41467-018-02926-5

O’Neil, S. M., Witcher, K. G., McKim, D. B., and Godbout, J. P. (2018). Forced turnover of aged microglia induces an intermediate phenotype but does not rebalance CNS environmental cues driving priming to immune challenge. Acta Neuropathol. Commun. 6:129. doi: 10.1186/s40478-018-0636-8

Palomer, E., Buechler, J., and Salinas, P. C. (2019). Wnt Signaling Deregulation in the Aging and Alzheimer's Brain. Front. Cell. Neurosci. 13:227. doi: 10.3389/ fncel.2019.00227

Paouri, E., and Georgopoulos, S. (2019). Systemic and CNS Inflammation Crosstalk: implications for Alzheimer's Disease. Curr. Alzheimer Res. 16, 559574. doi: 10.2174/1567205016666190321154618

Perry, V. H., Hume, D. A., and Gordon, S. (1985). Immunohistochemical localization of macrophages and microglia in the adult and developing mouse brain. Neuroscience 15, 313-326.

Pettigrew, L. C., Kryscio, R. J., and Norris, C. M. (2016). The TNFalpha-Transgenic Rat: hippocampal Synaptic Integrity, Cognition, Function, and Post-Ischemic Cell Loss. PLoS One 11:e0154721. doi: 10.1371/journal.pone.0154721

Pourbadie, H. G., Sayyah, M., Khoshkholgh-Sima, B., Choopani, S., Nategh, M., Motamedi, F., et al. (2018). Early minor stimulation of microglial TLR2 and TLR4 receptors attenuates Alzheimer's disease-related cognitive deficit in rats: behavioral, molecular, and electrophysiological evidence. Neurobiol. Aging 70, 203-216. doi: 10.1016/j.neurobiolaging.2018.06.020

Rangaraju, S., Dammer, E. B., Raza, S. A., Rathakrishnan, P., Xiao, H., Gao, T., et al. (2018). Identification and therapeutic modulation of a pro-inflammatory subset of disease-associated-microglia in Alzheimer's disease. Mol. Neurodegener. 13:24. doi: 10.1186/s13024-018-0254-8

Ransohoff, R. M. (2016). A polarizing question: do M1 and M2 microglia exist? Nat. Neurosci. 19, 987-991. doi: 10.1038/nn.4338

Rincon-Lopez, C., Tlapa-Pale, A., Medel-Matus, J. S., Martinez-Quiroz, J., Rodriguez-Landa, J. F., and Lopez-Meraz, M. L. (2017). Interleukin-1beta increases neuronal death in the hippocampal dentate gyrus associated with status epilepticus in the developing rat. Neurologia 32, 587-594. doi: 10.1016/j. nrl.2016.03.013

Rizzo, F. R., Musella, A., De Vito, F., Fresegna, D., Bullitta, S., Vanni, V., et al. (2018). Tumor Necrosis Factor and Interleukin-1beta Modulate Synaptic Plasticity during Neuroinflammation. Neural Plast. 2018:8430123. doi: 10. 1155/2018/8430123

Schneider, H., Pitossi, F., Balschun, D., Wagner, A., del Rey, A., and Besedovsky, H. O. (1998). A neuromodulatory role of interleukin-1beta in the hippocampus. Proc. Natl. Acad. Sci. U. S. A. 95, 7778-7783. doi: 10.1073/pnas.95.13.7778

Subhramanyam, C. S., Wang, C., Hu, Q., and Dheen, S. T. (2019). Microgliamediated neuroinflammation in neurodegenerative diseases. Semin. Cell Dev. Biol. 94, 112-120. doi: 10.1016/j.semcdb.2019.05.004

Taipa, R., das Neves, S. P., Sousa, A. L., Fernandes, J., Pinto, C., Correia, A. P., et al. (2019). Proinflammatory and anti-inflammatory cytokines in the CSF of patients with Alzheimer's disease and their correlation with cognitive decline. Neurobiol. Aging 76, 125-132. doi: 10.1016/j.neurobiolaging.2018.12.019

Tan, J., Town, T., Paris, D., Mori, T., Suo, Z., Crawford, F., et al. (1999). Microglial activation resulting from CD40-CD40L interaction after betaamyloid stimulation. Science 286, 2352-2355. doi: $10.1126 /$ science.286.5448. 2352

Tang, Y., and Le, W. (2016). Differential Roles of M1 and M2 Microglia in Neurodegenerative Diseases. Mol. Neurobiol. 53, 1181-1194. doi: 10.1007/ s12035-014-9070-5

Tang, Y., Li, T., Li, J., Yang, J., Liu, H., Zhang, X. J., et al. (2014). Jmjd3 is essential for the epigenetic modulation of microglia phenotypes in the immune pathogenesis of Parkinson's disease. Cell Death Differ. 21, 369-380. doi: 10.1038/cdd.2013. 159
Togo, T., Akiyama, H., Kondo, H., Ikeda, K., Kato, M., Iseki, E., et al. (2000). Expression of CD40 in the brain of Alzheimer's disease and other neurological diseases. Brain Res. 885, 117-121. doi: 10.1016/s0006-8993(00)02 984-X

van der Kant, R., Langness, V. F., Herrera, C. M., Williams, D. A., Fong, L. K., Leestemaker, Y., et al. (2019). Cholesterol Metabolism Is a Druggable Axis that Independently Regulates Tau and Amyloid-beta in iPSC-Derived Alzheimer's Disease Neurons. Cell Stem Cell 24, 363-375.e9. doi: 10.1016/j.stem.2018.12. 013

Veremeyko, T., Yung, A. W. Y., Dukhinova, M., Kuznetsova, I. S., Pomytkin, I., Lyundup, A., et al. (2018). Cyclic AMP Pathway Suppress Autoimmune Neuroinflammation by Inhibiting Functions of Encephalitogenic CD4 T Cells and Enhancing M2 Macrophage Polarization at the Site of Inflammation. Front. Immunol. 9:50. doi: 10.3389/fimmu.2018.00050

Walker, D. G., and Lue, L. F. (2015). Immune phenotypes of microglia in human neurodegenerative disease: challenges to detecting microglial polarization in human brains. Alzheimers Res. Ther. 7:56. doi: 10.1186/s13195-015-0 139-9

Wang, X., and Davis, R. L. (2021). Early Mitochondrial Fragmentation and Dysfunction in a Drosophila Model for Alzheimer's Disease. Mol. Neurobiol. 58, 143-155. doi: 10.1007/s12035-020-02107-w

Wang, Y., Cella, M., Mallinson, K., Ulrich, J. D., Young, K. L., Robinette, M. L., et al. (2015). TREM2 lipid sensing sustains the microglial response in an Alzheimer's disease model. Cell 160, 1061-1071. doi: 10.1016/j.cell.2015.01.049

Wang, Y., Zhu, T., Wang, M., Zhang, F., Zhang, G., Zhao, J., et al. (2019). Icariin Attenuates M1 Activation of Microglia and Abeta Plaque Accumulation in the Hippocampus and Prefrontal Cortex by Up-Regulating PPARgamma in Restraint/Isolation-Stressed APP/PS1 Mice. Front. Neurosci. 13:291. doi: 10. 3389/fnins.2019.00291

Wang, Z., Xu, Q., Cai, F., Liu, X., Wu, Y., and Song, W. (2019). BACE2, a conditional beta-secretase, contributes to Alzheimer's disease pathogenesis. JCI Insight 4:e123431. doi: 10.1172/jci.insight.123431

Wu, J., Ding, D. H., Li, Q. Q., Wang, X. Y., Sun, Y. Y., and Li, L. J. (2019). Lipoxin A4 Regulates Lipopolysaccharide-Induced BV2 Microglial Activation and Differentiation via the Notch Signaling Pathway. Front. Cell. Neurosci. 13:19. doi: 10.3389/fncel.2019.00019

Xiao, J., Yao, R., Xu, B., Wen, H., Zhong, J., Li, D., et al. (2020). Inhibition of PDE4 Attenuates TNF-alpha-Triggered Cell Death Through Suppressing NF-kappaB and JNK Activation in HT-22 Neuronal Cells. Cell. Mol. Neurobiol. 40, 421-435. doi: 10.1007/s10571-019-00745-w

Yang, G., Parkhurst, C. N., Hayes, S., and Gan, W. B. (2013). Peripheral elevation of TNF-alpha leads to early synaptic abnormalities in the mouse somatosensory cortex in experimental autoimmune encephalomyelitis. Proc. Natl. Acad. Sci. U. S. A. 110, 10306-10311. doi: 10.1073/pnas.122289 5110

Yang, L., Lindholm, K., Konishi, Y., Li, R., and Shen, Y. (2002). Target depletion of distinct tumor necrosis factor receptor subtypes reveals hippocampal neuron death and survival through different signal transduction pathways. J. Neurosci. 22, 3025-3032. doi: 10.1523/JNEUROSCI.22-08-03025.2002

Yiangou, Y., Facer, P., Durrenberger, P., Chessell, I. P., Naylor, A., Bountra, C., et al. (2006). COX-2, CB2 and P2X7-immunoreactivities are increased in activated microglial cells/macrophages of multiple sclerosis and amyotrophic lateral sclerosis spinal cord. BMC Neurol. 6:12. doi: 10.1186/1471-2377-6-12

Yin, Z., Raj, D., Saiepour, N., Van Dam, D., Brouwer, N., Holtman, I. R., et al. (2017). Immune hyperreactivity of Abeta plaque-associated microglia in Alzheimer's disease. Neurobiol. Aging 55, 115-122. doi: 10.1016/j. neurobiolaging.2017.03.021

Yoder, M. C. (2014). Inducing definitive hematopoiesis in a dish. Nat. Biotechnol. 32, 539-541. doi: 10.1038/nbt.2929

Zhang, X., He, Q., Huang, T., Zhao, N., Liang, F., Xu, B., et al. (2019). Treadmill Exercise Decreases Abeta Deposition and Counteracts Cognitive Decline in APP/PS1 Mice, Possibly via Hippocampal Microglia Modifications. Front. Aging Neurosci. 11:78. doi: 10.3389/fnagi.2019.00078

Zhang, Z. J., Zheng, X. X., Zhang, X. Y., Zhang, Y., Huang, B. Y., and Luo, T. (2019). Aging alters Hvl-mediated microglial polarization and enhances neuroinflammation after peripheral surgery. CNS Neurosci. Ther. 26, 374-384. doi: $10.1111 / \mathrm{cns} .13271$ 
Zhang, Y., Dong, Z., and Song, W. (2020). NLRP3 inflammasome as a novel therapeutic target for Alzheimer's disease. Signal Transduct. Target. Ther. 5:37. doi: 10.1038/s41392-020-0145-7

Zhang, Y., Feng, S., Nie, K., Li, Y., Gao, Y., Gan, R., et al. (2018). TREM2 modulates microglia phenotypes in the neuroinflammation of Parkinson's disease. Biochem. Biophys. Res. Commun. 499, 797-802. doi: 10.1016/j.bbrc. 2018.03.226

Zhang, Y., and He, M. L. (2017). Deferoxamine enhances alternative activation of microglia and inhibits amyloid beta deposits in APP/PS1 mice. Brain Res. 1677, 86-92. doi: 10.1016/j.brainres.2017.09.019

Zhao, L. (2019). CD33 in Alzheimer's Disease - Biology, Pathogenesis, and Therapeutics: a Mini-Review. Gerontology 65, 323-331. doi: 10.1159/ 000492596

Zhou, J., Zhang, X., Zhou, Y., Wu, B., and Tan, Z. Y. (2019). Up-regulation of P2X7 Receptors Contributes to Spinal Microglial Activation and the Development of Pain Induced by BmK-I. Neurosci. Bull. 35, 624-636. doi: 10.1007/s12264-01900345-0

Zhou, X., Wahane, S., Friedl, M. S., Kluge, M., Friedel, C. C., Avrampou, K., et al. (2020). Microglia and macrophages promote corralling, wound compaction and recovery after spinal cord injury via Plexin-B2. Nat. Neurosci. 23, 337-350. doi: 10.1038/s41593-020-0597-7

Conflict of Interest: The authors declare that the research was conducted in the absence of any commercial or financial relationships that could be construed as a potential conflict of interest.

Publisher's Note: All claims expressed in this article are solely those of the authors and do not necessarily represent those of their affiliated organizations, or those of the publisher, the editors and the reviewers. Any product that may be evaluated in this article, or claim that may be made by its manufacturer, is not guaranteed or endorsed by the publisher.

Copyright (c) 2021 Wang, Yao, Liu, Ya, Cheng, Xing and Wu. This is an open-access article distributed under the terms of the Creative Commons Attribution License (CC BY). The use, distribution or reproduction in other forums is permitted, provided the original author(s) and the copyright owner(s) are credited and that the original publication in this journal is cited, in accordance with accepted academic practice. No use, distribution or reproduction is permitted which does not comply with these terms. 